BULL. AUSTRAL. MATH. SOC.

$54 \mathrm{E} 40, \quad 54 \mathrm{H} 25$

VOL. 29 (1984), 349-356.

(47 H10)

\title{
GENERALIZATION OF LEADER'S FIXED POINT PRINCIPLE
}

\author{
TANMOY SOM AND R, N. MukHerJee
}

In J. Math. Anal. Appl. 61 (1977), 466-474, Leader has given a fixed point principle for an operator $f: X \rightarrow X$, where $X$ is a metric-space, based on conditional uniform equivalence of orbits. We generalize this principle for two mappings $f_{1}$ and $f_{2}$ to give common fixed point results in two different ways. Further we derive an $f$-generalized fixed point theorem for two commuting mappings.

\section{Introduction}

Let $\left(f_{Z}, X, d\right), \quad=1,2$, be two operators where $f_{Z}: X \rightarrow X$ and $X$ is a metric space. Let $F \cong f_{2} f_{1}$ be the composite map of $f_{1}$ and $f_{2}$. Then we define the generalized orbit of a point $x \in X$ as the sequence of iterates $x, f_{1}(x), f_{2} f_{1}(x) \cong F(x), f_{1} F(x), F^{2}(x)$, $f_{1} F^{2}(x), F^{3}(x), \ldots$. The limit $p$ of a generalized convergent orbit must be fixed under certain weak conditions (for example, $f_{1}, f_{2}$ have closed graphs or $d\left(x, f_{l}(x)\right), 2=1,2$, is lower semicontinuous), that is, $p=f_{l}(p), \quad l=1,2$. We call a fixed point $p$ a generalized fixed

Received 2 December 1983.

Copyright Clearance Centre, Inc. Serial-fee code: 0004-9727/84 $\$$ A2.00 +0.00 . 
point if it is the limit of every generalized orbit in $X$. A necessary condition for a fixed point is the equivalence of all generalized orbits. Now we give our first result as follows.

THEOREM 1. Let $\left(f_{l}, X, d\right), Z=1,2$, be two operators on $a$ metric space $(X, d)$. Let $\left\{x_{n}\right\}$ and $\left\{y_{n}\right\}$ be two sequences generated by generalized orbits of $x_{0}$ and $y_{0}$ respectively with the applications of maps $f_{1}$ and $f_{2}$ in the following way:

$$
x_{1}=f_{1}\left(x_{0}\right), x_{2}=f_{2}\left(x_{1}\right), \cdots \text {, }
$$

and in general

$$
x_{2 n+1}=f_{1}\left(x_{2 n}\right), x_{2 n+2}=f_{2}\left(x_{2 n+1}\right), n=0,1,2, \ldots,
$$

and

$$
y_{1}=f_{2}\left(y_{0}\right), y_{2}=f_{1}\left(y_{1}\right), \ldots,
$$

and in general

$$
y_{2 n+1}=f_{2}\left(y_{2 n}\right), y_{2 n+2}=f_{1}\left(y_{2 n+1}\right), n=0,1,2, \ldots
$$

Let $\varepsilon_{i}, \quad i \in N$, be positive real numbers defined by

$$
\varepsilon_{n}=\sup \left\{d\left(x_{i}, y_{i}\right): i \geq n, d\left(x_{0}, y_{0}\right) \leq c\right\} \text {. }
$$

If $(m / 2)\left(\varepsilon_{n}+\varepsilon_{n+1}\right)+\frac{1}{2}\left(\varepsilon_{n}-\varepsilon_{n+1}\right)+\varepsilon_{m} \leq c$ and $d\left(x, f_{2}(x)\right) \leq c$,

$\tau=1,2$, then, for all $i \geq n$ and all $j$ in $N$,

$$
d\left(x_{i}, x_{i+j}\right) \leq \frac{m}{2}\left(\varepsilon_{n}+\varepsilon_{n+1}\right)+\frac{1}{2}\left(\varepsilon_{n}-\varepsilon_{n+1}\right)+\varepsilon_{m} .
$$

Further if

$$
d\left(x_{n}, y_{n}\right)+0
$$

uniformly for all $x_{0}, y_{0}$ in $x$ with $d\left(x_{0}, y_{0}\right) \leq c$ then

$$
\text { the sequence }\left\{x_{n}\right\} \text { is uniformly Cauchy. }
$$

If the graphs of both $\left(f_{\eta}, X, d\right), Z=1,2$, are complete and (3) holds then $d\left(x, f_{l}(x)\right) \leq c, \quad l=1,2$, implies that $f_{1}$ and $f_{2}$ have a 
common fixed point.

Proof. By the induction process on $k$ we shall prove (2) for $j \leq k m$ for all $k$ in $N$ under the given condition that, for a given $m, n$,

$$
\frac{m}{2}\left(\varepsilon_{n}+\varepsilon_{n+1}\right)+\frac{1}{2}\left(\varepsilon_{n}-\varepsilon_{n+1}\right)+\varepsilon_{m} \leq c
$$

and

$$
d\left(x, f_{l}(x)\right) \leq c, \quad \imath=1,2 \text { for all } x \in X
$$

Let $x_{i}=(x)_{i}$, that is, $i$ iterations on initial point $x$ by $f_{1}$ and $f_{2}$ accordingly. Let $k=1$, that is, $j \leq m$; then (1) implies for all $i \geq n$ and $j \leq m, m$ even, that

$$
\text { (5) } \begin{aligned}
d\left(x_{i}, x_{i+j}\right) \leq & d\left(x_{i}, x_{i+1}\right)+d\left(x_{i+1}, x_{i+2}\right)+\ldots+d\left(x_{i+j-1}, x_{i+j}\right) \\
\leq & d\left(\left(x_{0}\right)_{i},\left(x_{1}\right)_{i}\right)+d\left(\left(x_{0}\right)_{i+1},\left(x_{1}\right)_{i+1}\right)+d\left(\left(x_{2}\right)_{i},\left(x_{3}\right)_{i}\right) \\
& \quad+d\left(\left(x_{2}\right)_{i+1},\left(x_{3}\right)_{i+1}\right)+\ldots+d\left(\left(x_{j-2}\right)_{i+1},\left(x_{j-1}\right)_{i+1}\right) \\
& \leq \varepsilon_{n}+\varepsilon_{n+1}+\varepsilon_{n}+\varepsilon_{n+1}+\ldots+\varepsilon_{n}+\varepsilon_{n+1} \\
& \leq \frac{j}{2}\left(\varepsilon_{n}+\varepsilon_{n+1}\right) \leq \frac{m}{2}\left(\varepsilon_{n}+\varepsilon_{n+1}\right) .
\end{aligned}
$$

When $m$ is odd we get

$$
\begin{aligned}
d\left(x_{i}, x_{i+j}\right) & \leq d\left(x_{i}, x_{i+1}\right)+\ldots+d\left(x_{i+j-1}, x_{i+j}\right) \\
& \leq d\left(\left(x_{0}\right)_{i},\left(x_{1}\right)_{i}\right)+d\left(\left(x_{0}\right)_{i+1},\left(x_{1}\right)_{i+1}\right) \\
& \quad+\ldots+d\left(\left(x_{j-3}\right)_{i+1},\left(x_{j-2}\right)_{i+1}\right)+d\left(\left(x_{j-1}\right)_{i},\left(x_{j}\right)_{i}\right) \\
& \leq \varepsilon_{n}+\varepsilon_{n+1}+\varepsilon_{n}+\ldots+\varepsilon_{n+1}+\varepsilon_{n} \\
& =\left(\frac{j+1}{2}\right) \varepsilon_{n}+\left(\frac{j-1}{2}\right) \varepsilon_{n+1} \\
& \leq \frac{m}{2}\left(\varepsilon_{n}+\varepsilon_{n+1}\right)+\frac{1}{2}\left(\varepsilon_{n}-\varepsilon_{n+1}\right) .
\end{aligned}
$$

Thus

$$
d\left(x_{i}, x_{i+j}\right) \leq \frac{m}{2}\left(\varepsilon_{n}+\varepsilon_{n+1}\right)+\frac{1}{2}\left(\varepsilon_{n}-\varepsilon_{n+1}\right)
$$

for all $i \geq n$ and $j \leq m$, independent of $m$ even or odd, that is, (2) holds for all $j \leq m$. Now we suppose that (2) holds for all $j \leq k m$ and prove it for $j \leq(k+1)_{m}$. Consider $k m<j \leq(k+1) m$. Then $0<j-m \leq k m$ 
and so our induction process gives

$$
d\left(x_{i}, x_{i+j-m}\right) \leq \frac{m}{2}\left(\varepsilon_{n}+\varepsilon_{n+1}\right)+\frac{1}{2}\left(\varepsilon_{n}-\varepsilon_{n+1}\right)+\varepsilon_{m} \leq c
$$

for all $i \geq n$. Now iterating $m$ times by $f_{1}$ and $f_{2}$ accordingly the above we get

$$
d\left(x_{i+m}, x_{i+j}\right) \in \varepsilon_{m} \text { for all } i \geq n
$$

here $x_{0}=x_{i}$ and $y_{0}=x_{i+j-m}$.

Therefore from (6) with $j=m$ and (7) we get

$$
\begin{aligned}
d\left(x_{i}, x_{i+j}\right) & \leq d\left(x_{i}, x_{i+m}\right)+d\left(x_{i+m}, x_{i+j}\right) \\
& \leq \frac{m}{2}\left(\varepsilon_{n}+\varepsilon_{n+1}\right)+\frac{1}{2}\left(\varepsilon_{n}-\varepsilon_{n+1}\right)+\varepsilon_{m},
\end{aligned}
$$

for all $i \geq n$. Thus (2) is true for all $j \leq(k+1) m$ and hence for all $j$ in $N$.

Now from (1) and (3) we have $\varepsilon_{n} \downarrow 0$. Therefore for a given $0<\varepsilon<c$, we can choose $m$ so large that $\varepsilon_{m}<\varepsilon$. Further we take $n$ so large that

$$
\left(\varepsilon_{n}+\varepsilon_{n+1}\right)+m^{-1}\left(\varepsilon_{n}-\varepsilon_{n+1}\right)<2 m^{-1}\left(\varepsilon-\varepsilon_{m}\right) \text {. }
$$

(For this we choose $n$ large enough so that $\varepsilon_{n}<\left(\varepsilon-\varepsilon_{m}\right) / m$ and this gives

$$
\left(1+\frac{1}{m}\right) \varepsilon_{n}+\left(1-\frac{1}{m}\right) \varepsilon_{n}<2 \frac{\left(\varepsilon-\varepsilon_{m}\right)}{m}
$$

or

$$
\left(1+\frac{1}{m}\right) \varepsilon_{n}+\left(1-\frac{1}{m}\right) \varepsilon_{n+1}<2 \frac{\left(\varepsilon-\varepsilon_{m}\right)}{m}
$$

which consequently satisfies (8)). Thus we have

$$
\frac{m}{2}\left(\varepsilon_{n}+\varepsilon_{n+1}\right)+\frac{1}{2}\left(\varepsilon_{n}-\varepsilon_{n+1}\right)+\varepsilon_{m}<\varepsilon<c .
$$

So (2) holds for all $j$ in $N$ and hence $a\left(x_{i}, x_{i+j}\right)<\varepsilon$ for all $i \geq n$ and $j$ in $N$ and all $x$ with $d\left(x, f_{l}(x)\right) \leq c$, which proves (4). 
Further let the graphs of $\left(f_{l}, X, d\right), Z=1,2$, be complete and (3) hold, then for all $x$ with $d\left(x, f_{l}(x)\right) \leq c, Z=1,2$, (4) gives that $\left\{f_{1}\left(x_{2 n}\right)\right\}$ and $\left\{f_{2}\left(x_{2 n+1}\right)\right\}$ are Cauchy and hence by graphcompleteness we have

$$
f_{1}\left(x_{2 n}\right) \rightarrow f_{1}(t) \text { with } x_{2 n} \rightarrow t
$$

and

$$
f_{2}\left(x_{2 n+1}\right) \rightarrow f_{2}(t) \text { with } x_{2 n+1} \rightarrow t, \quad t \in X .
$$

Therefore $f_{1}(t)=t$ and $f_{2}(t)=t$, that is, $f_{I}(t)=f_{2}(t)=t$ which completes the whole proof.

REMARK. In case $f_{1}=f_{2}=f$ we get Theorem 1 of Leader [1] as a corollary. (In that case $\varepsilon_{n+1}$ reduces to $\varepsilon_{n}$. )

In what follows we give another generalization of Leader's fixed point principle by considering a composite map.

THEOREM 2. Let $\left(f_{l}, X, d\right), Z=1,2$, be two operators on a metric space $(X, d)$. Let, for any $x_{0} \in X,\left\{x_{n}\right\}$ be a sequence as defined in Theorem 1. Now let $\left\{x_{2 n}\right\}$ be a sequence generated by the composite map $F \cong f_{2} f_{1}$ in the following way with $x_{0}$ as the starting point:

$$
x_{2}=F\left(x_{0}\right), \quad x_{4}=F\left(x_{2}\right)=F^{2}\left(x_{0}\right) \ldots
$$

and in general, $x_{2 n+2}=F\left(x_{2 n}\right)=F^{n+1}\left(x_{0}\right), n=0,1,2, \ldots$. Now we define real numbers $\varepsilon_{2 i}, \quad i \in N$, for some $c>0$ as

$$
\varepsilon_{2 n}=\sup \left\{d\left(F^{i} x_{0}, F^{i} y_{0}\right): i \geq n, d\left(x_{0}, y_{0}\right) \leq c\right\}
$$

where $\left\{y_{2 n}\right\}$ is a sequence generated by repeated application of $F$ on $y_{0}$. If $m \varepsilon_{2 n}+\varepsilon_{2 m} \leq c$ and $d(x, F(x)) \leq c$, then

$$
d\left(x_{2 i}, x_{2 i+2 j}\right) \leq m \varepsilon_{2 n}+\varepsilon_{2 m} \text { for all } i \geq n
$$


and alz $j$ in $N$. Hence if

$$
d\left(x_{2 n}, y_{2 n}\right) \rightarrow 0
$$

uniformly for all $x_{0}, y_{0}$ in $x$ with $d\left(x_{0}, y_{0}\right) \leq c$, then

$$
\text { the sequence }\left\{x_{2 n}\right\} \text { is uniformly Cauchy. }
$$

Again if the groph of $(F, X, d)$ is complete and (11) holds then $d(x, F(x)) \leq c$ implies that $F$ has a fixed point. Further if

$$
d(F(x), F(y))<d(x, y),
$$

then $F$ has a unique fixed point. Now if $f_{1} f_{2}=f_{2} f_{1}$ then the fixed point of $F$ becomes the unique common fixed point of $f_{1}$ and $f_{2}$.

The proof of (10), (12) and that $F$ has a fixed point follows the same line as that of Theorem 1 of Leader [1]. Further, condition (13) guarantees the uniqueness of the fixed point of $F$ which, on combination with the commutativity of $f_{1}$ and $f_{2}$, gives the existence of the unique common fixed point (same as that of $F$ ) of both the maps and hence the result.

THEOREM 3. Let $(X, d)$ be a metric space. Let $f$ and $g$ be two mappings of $X$ into $X$ with $f$ continuous. Let $f$ and $g$ commute with each other with $g(X) \subset f(X)$. For some $x_{0} \in X$ we define a sequence $\left\{y_{n}\right\}$ as folzows:

$$
y_{1}=f\left(x_{1}\right)=g\left(x_{0}\right), y_{2}=f\left(x_{2}\right)=g\left(x_{1}\right), \cdots
$$

and

$$
y_{n}=f\left(x_{n}\right)=g\left(x_{n-1}\right), n=1,2, \ldots .
$$

Simizarly, for some $p_{0} \in X$, let us have a sequence $\left\{z_{n}\right\}$, that is, $z_{n}=f\left(p_{n}\right)=g\left(p_{n-1}\right), n=1,2, \ldots$. For some $c>0$, define

$$
\varepsilon_{n}=\sup \left\{d\left(y_{i}, z_{i}\right): i \geq n-1, d\left(g\left(x_{0}\right), g\left(p_{0}\right)\right) \leq c\right\} .
$$

If $m \varepsilon_{n}+\varepsilon_{m} \leq c$ and $d(f(x), g(x)) \leq c$ then

$$
d\left(y_{i}, y_{i+j}\right) \leq m \varepsilon_{n}+\varepsilon_{m} \text { for all } i \geq n-1
$$


and alz $j$ in $N$. Hence if

$$
d\left(y_{n}, z_{n}\right) \rightarrow 0
$$

uniformly for all $x_{0}, p_{0} \in X$ with $d\left(g\left(x_{0}\right), g\left(p_{0}\right)\right) \leq c$ then the sequence $\left\{y_{n}\right\}$ is uniformly Cauchy, and further if $g$ satisfies

$$
d(g(x), g(y)) \leq d(f(x), f(y)) \text { for alz } x, y \in X,
$$

then $g$ has an f-generalized fixed point.

Before giving the proof of the theorem we give the definition of an $f$-generalized fixed point.

DEFINITION. Let $f, g:(X, d) \rightarrow(X, d)$ be two maps with $g(X) \subset f(X)$. If there exists a point $t \in X$ such that $g(t)=f(t)$ then we say that $g$ has an $f$-generalized fixed point.

Proof. The proof of (15) and that $\left\{y_{n}\right\}$ is uniformly Cauchy goes in a similar fashion as that of Theorem 1 of Leader [1], so we omit it.

Let $y_{n} \rightarrow t$. Since $f$ is continuous we have $f\left(y_{n}\right) \rightarrow f(t)$. Now (17) gives that $d\left(g\left(y_{n}\right), g(t)\right) \leq d\left(f\left(y_{n}\right), f(t)\right)$, which in the limiting case implies $g\left(y_{n}\right) \rightarrow g(t)$.

Thus $f\left(y_{n}\right)=f\left(g\left(x_{n-1}\right)\right)=g\left(f\left(x_{n-1}\right)\right)=g\left(y_{n-1}\right) \rightarrow g(t)$. Therefore $f(t)=g(t)$. Hence $g$ has an $f$-generalized fixed point $t$. In case $f$ is an identity map we get an ordinary fixed point.

THEOREM 4. Let $\left(f_{l}, x, d\right), l=1,2$, be two operators with graphs of $f_{2}$ both complete. Let for some $c>0,(X, d)$ be weakly c-chained and (3) hold. Then $f_{1}, f_{2}$ have a common fixed point.

Proof. Let $\left\{x_{n}\right\}$ and $\left\{y_{n}\right\}$ be two sequences as defined in Theorem 1 with initial points $x_{0}$ and $y_{0}$ respectively. Since $(X, d)$ is weakly $c$-chained (see Leader [1], Theorem 2), we have for any $x, y \in X$ a finite sequence $\left\langle x^{0}, x^{1}, \ldots, x^{m}\right\rangle$ with $x^{0}=x, x^{m}=y$ and $d\left(x^{i}, x^{i+1}\right) \leq c$ for $i=0,1, \ldots, m-1$. Then in $x_{n}, y_{n}$ applying triangle inequality we have 


$$
\begin{aligned}
d\left(x_{n}, y_{n}\right) & =d\left(x_{n}^{0}, x_{n}^{m}\right) \\
& \leq d\left(x_{n}^{0}, x_{n}^{1}\right)+d\left(x_{n}^{1}, x_{n}^{2}\right)+\ldots+d\left(x_{n}^{m-1}, x_{n}^{m}\right),
\end{aligned}
$$

where $d\left(x_{n}^{i}, x_{n}^{i+1}\right) \leq c$, for $i=0,1, \ldots, m-1$.

By using similar arguments as in proving (5) or (6) in the above and then applying (3) we get $d\left(x_{n}, y_{n}\right) \rightarrow 0$, as $n \rightarrow \infty$ for all $x, y \in X$ which further implies that all generalized orbits are equivalent.

In case $y=f_{1}\left(x_{0}\right)$, we get

$$
d\left(x_{n}, f_{1}\left(x_{n}\right)\right) \rightarrow 0, n \text { even, }
$$

and

$$
d\left(x_{n}, f_{2}\left(x_{n}\right)\right) \rightarrow 0, n \text { odd }
$$

Therefore for $n$ large enough we have $d\left(x_{n}, f_{l}\left(x_{n}\right)\right)<c, l=1,2$, accordingly with $n$ even or $n$ odd. Hence Theorem 1 gives the result.

THEUREM 5. Let $(F, X, d)$, where $F=f_{2} f_{1}$, be a composite operator as defined in Theorem 2 with the groph of $(F, X, d)$ complete. Let for some $c>0,(X, d)$ be weakly c-chained and (11) hold. Then $F$ has a fixed point.

Proof. The proof of the above theorem is analogous to that of the above theorem, so we omit it.

\section{References}

[1] S. Leader, "Fixed points for operators on metric spaces with conditional uniform equivalence of orbits", J. Math. Anal. Appl. 61 (1977), 466-474.

[2] A. Meir and E. Keeler, "A theorem on contraction mappings", J. Math. Anal. Appl. 28 (1969), 326-329.

Applied Mathematics Section, School of Applied Sciences, Institute of Technology, Banaras Hindu University, Varanasi 221005, India. 